\title{
Study on the preparation and properties of UV curable polyurethane materials modified by organic silicon
}

\author{
Xidan Luo ${ }^{1}$, Xuetao $\mathrm{He}^{2}$, Pengcheng $\mathrm{Xie}^{3}$, Jianyun $\mathrm{He}^{4}$, a , Yumei Ding ${ }^{5}$ and Bingqian Xia ${ }^{6}$ \\ ${ }^{1}$ Beijing, Chaoyang District North Third Ring Road No 15, Beijing University of Chemical Technology, China. \\ This study was supported by the National Natural Science Foundation of China (NO.51573017)
}

\begin{abstract}
In this paper, polyester polyol, toluene diisocyanate (TDI) and hydroxyethyl acrylate (HEA) were taken as the raw materials to synthesize polyurethane acrylate. UV curable organic silicon nano sol was synthesized by $\gamma$ methacryloxypropyltrime-thoxysilane. The UV curable polyurethane materials modified by organic silicon were prepared based on the UV curable organic silicon nano sol and synthesized polyurethane acrylate. This paper studies on the mechanical properties of organic silicon modified UV curable polyurethane materials and puts forward the micro model that inorganic phase is bonded to the resin in the form of chemical bonds. The results show that the heat resistance and mechanical properties of hybrid materials are improved with the increase of the nano silica sol's contents, the abrasion resistance of hybrid coatings is also improved and the silica nano particles are well dispersed in the organic phase.
\end{abstract}

\section{Introduction}

UV curable technology is a method to form a curable coating and products by taking ultraviolet light as curing energy. Photo-chemical reactions occurred in this process when liquid UV materials are excited by the UV light. UV curable technology has many advantages such as quickcuring, high-efficiency, energy saving, environmental protection, excellent performance of the cured product and so on. It is an environmentally friendly and energy efficient technology ${ }^{[1-2]}$. Polyurethane acrylate is an important light curing oligomer, which has high toughness and oil resistance after curing while the heat resistance is poor.

Organic / inorganic hybrid material will have excellent performance because organic / inorganic hybrid material could combine the characteristics of high toughness, high elasticity, excellent machinability of the organic phase with the high hardness and high modulus performance of the inorganic phase. It has become a research focus due to its obvious advantages in many aspects ${ }^{[3-6]}$.

The technology that prepares UV curable nano silica sol by sol-gel method has a lot of advantages.The mild reaction condition is a kind of this advantages, it means the synthesis can realize at room temperature or slightly above the room temperature. In recent years, sol-gel technique has been rapidly developed in the preparation of organic / inorganic hybrid materials.

KeChangme ${ }^{\mathrm{i}[7]}$ prepared polyacrylate/TiO2 - $\mathrm{SiO} 2$ nano hybrid material with butyl titanate and ethyl orthosilicate as the metal alkoxide precursor, the appearance of inorganic particle in hybrid material is spherical, particle size is $90 \sim 110 \mathrm{~nm}$. Wei ${ }^{[8]}$ finished the 
hydrolyzed ethyl silicatecondensation in the presence of hydroxy ethyl methacrylate and prepared hybrid materials that inorganic phase and the organic phase were combined though covalent bond. Yuan Jian prepared polyvinyl acetate/silica.

\section{Experimental part}

\subsection{The main materials}

Polyester polyol used in this experiment is provided by Qingdao Yu Tian New Material Co.LTD. Toluene diisocyanate (TDI) is bought from Tianjin chemical reagent factory, Hydroxyethyl acrylate (HEA) is bought from Beijing Dongfang acrylic Chemical Technology Co. LTD, Di-butyltin dilaurate (DBTDL) is bought from Beijing Zheng heng Chemical Co.LTD, Tripropylene glycol diacrylate(TPGDA) is bought from Tianjiao chemicalcompany of Tianjin city. The 2- hydroxy -2 methyl -1 phenyl -1 acetone (Darocur1173) is provided by Ciba Geigy, $\gamma$-methacryloxypropyltrimethoxysilane is bought from Nanjing Nengde chemical Co.LTD and the 37\% hydrochloric acid, absolute alcohol are bought from Beijing chemical reagent factory.

\subsection{The main instruments and equipments}

The main instruments used in the tests are: UV-curing machine RW - UVA 201-20, Video based contact angle

Measuring device OCAZO, thermogravimetric analyzer TG209C , Electronic Universal Material Testing Machine LR30KPlu, Differential scanning calorimetry DSC204F1, thermostat water bath HHS11-Ni1, Precision timing electric mixer JJ-1 and other major equipment.

\subsection{Performance characterization}

\subsubsection{Infrared spectrum analysis}

The infrared spectra of nano sol was tested through the $\mathrm{KBr}$ tabletting method by Fourier transform infrared spectrometer tensor27 type that is produced by Bruker Corporation of Germany, scanning range is $400 \mathrm{~cm}^{-1} \sim 4000$ $\mathrm{cm}^{-1}$.

\subsubsection{Thermogravimetric analysis (TGA)}

The speed of heating is $20^{\circ} \mathrm{C} / \mathrm{min}$, temperature range from $30^{\circ} \mathrm{C}$ to $600^{\circ} \mathrm{C}$ and test the concentration of nitrogen.

\subsubsection{Coating hardness test}

The pencil hardness of the coating according to the national standard GB6739-86 is done by using a set of pencils whose hardness is known. The hardness of the coating is expressed as the depth that pencil core can penetrate the surface of the coating to substrate.

\subsubsection{Coating adhesion test}

For adhesion test, the QFH Paints-cross-cut tester is used, according to the determination of GB/T1720-1979. Test results are divided into six grades. Among them level 0 is for the best, while 5 is for the worst.

\subsection{Synthesis of polyurethane acrylate}

1)Put a certain amount of polyester polyol 2356 into three mouth flask equipped with a stirrer, a condenser pipe and a thermometer,then evacuate the flask for 1 hour at $110{ }^{\circ} \mathrm{C}$.

2)Cool it until room temperature, then add toluene diisocyanate (TDI) , add several drops of di-butyltin dilaurate (DBTDL) at the same time, stir it and warm it up to $30 \sim 35^{\circ} \mathrm{C}$ gradually,stop heating and let it self heating. When the temperature reached $68 \sim 70{ }^{\circ} \mathrm{C}$, react for 1.5 hours under this temperature, then react further for 1.5 hours at $75^{\circ} \mathrm{C}$.

3)Put a certain amount of Hydroxyethyl acrylate (HEA), then add several drops of di-butyltin dilaurate (DBTDL) and inhibitor (HEMQ) at the same time, react for 1.5 hours at $75^{\circ} \mathrm{C}$, cool it down to $50^{\circ} \mathrm{C} \sim 60^{\circ} \mathrm{C}$.

4)Add the diluent tripropylene glycol diacrylate (TPGDA), stir for a period of time and the resin of polyurethane acrylate was synthesized. 


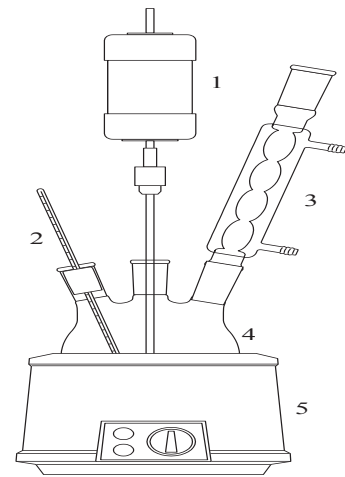

Fig. 1 Device for the synthesis of polyurethane acrylate (PUA) 1-stirrer 2-thermometer 3-condenser pipe 4-three mouth

flask equipped 5 -Heating jacket

\subsection{Methods for synthesis of nano sol}

A certain amount of anhydrous ethanol, $\gamma$ methacryloxypropyltrime-thoxysilane and $3.6 \mathrm{~g}$ of distilled water were put into three mouth flask equipped with a stirrer, a condenser pipe and a thermometer, drop in anhydrous ethanol and $4 \mathrm{ml}$ of hydrochloric acid mixture, let the $\mathrm{pH}$ value of the solution become 2 , and uniformly stir it at room temperature for 20 minutes, then heat it up to $65^{\circ} \mathrm{C}$, react for two hours, cool the solution and add the trimethylchlorosilane which is called capping agent, react half an hour. Finally, make the decompression and remove the solvent at $60{ }^{\circ} \mathrm{C}$, cool it and get the UV-curable nano sol products.

\subsection{The preparation of UV-curable hybrid coatings}

A certain concentration of nano sol and photoinitiator 1173 was put into the polyurethane acrylate, then mixed homogeneously to get UV-curable hybrid coating.

Table 1. Formula of UV-curable hybrid coating

\begin{tabular}{|c|c|c|}
\hline Hybrid coating & Sol (wt\%) & $\begin{array}{c}\text { Light Initiator } \\
(\mathrm{wt} \%)\end{array}$ \\
\hline $1^{\#}$ & 0 & 4 \\
$2^{\#}$ & 5 & 4 \\
$3^{\#}$ & 10 & 4 \\
$4^{\#}$ & 15 & 4 \\
$5^{\#}$ & 20 & 4 \\
\hline
\end{tabular}

\section{Results and discussion}

\subsection{Infrared spectrum analysis of the synthesized polyurethane acrylate}

Figure 2 is the FT-IR spectra of polyurethane acrylate, we can learn from the picture that the peak near $3379 \mathrm{~cm}^{-1}$ and $1525 \mathrm{~cm}^{-1}$ is the stretching vibration band of $-\mathrm{NH}-$, the peak near $1721 \mathrm{~cm}^{-1}$ is the stretching vibration band of $\mathrm{C}=\mathrm{O}$. The stretching vibration bands of $-\mathrm{NH}-$ and $\mathrm{C}=\mathrm{O}$ indicate the formation of polyurethane. The peak near $2927 \mathrm{~cm}^{-1}$ is the stretching vibration band of $-\mathrm{CH}_{2}-$. The behavior that most of the stretching vibration band of - NCO near 2275 2240 $\mathrm{cm}^{-1}$ disappear shows the group of $-\mathrm{NCO}$ has been reacted basically. The stretching vibration bands of $-\mathrm{C}=\mathrm{C}-$ near $1636 \mathrm{~cm}^{-1}$ and $810 \mathrm{~cm}^{-}$ ${ }^{1}$ indicate that double bond of acrylate has been linked on the polyurethane molecular chain. The peak near $1460 \mathrm{~cm}^{-}$ ${ }^{1}$ is the deformation vibration peak of $-\mathrm{CH}_{2}-$ and the peak near $1409 \mathrm{~cm}^{-1}$ is the stretching vibration band of H

$\mathrm{C}=\mathrm{CH}_{2}$, the peak near $776 \mathrm{~cm}^{-1}$ is the stretching vibration band of benzene ring.

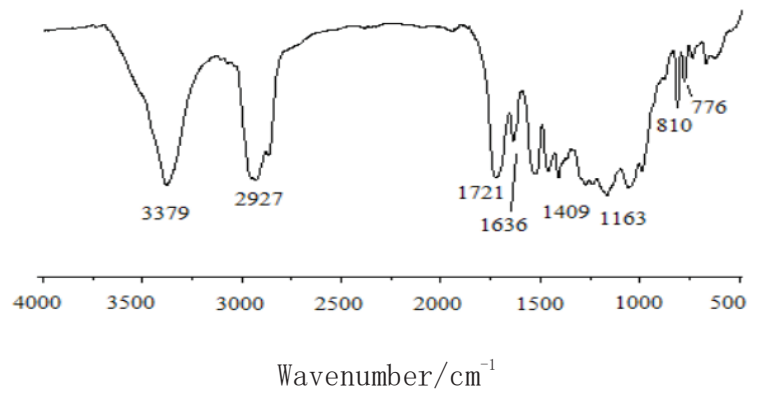

Fig. 2 FT-IR spectrum of PUA

\subsection{The analysis of the infrared spectrum of hybrid coating}

Figure 3 is the FT-IR spectra of UV curable nano sol /PUA hybrid coatings. The picture shows that the peaks near $3372 \mathrm{~cm}^{-1}$ and $1530 \mathrm{~cm}^{-1}$ are the stretching vibration band of $-\mathrm{NH}-$, the peaks near $2951 \mathrm{~cm}^{-1}$ and $1722 \mathrm{~cm}^{-1}$ are the stretching vibration band of $-\mathrm{C}-\mathrm{H}$ and $-\mathrm{COO}$ - , the stretching vibration band of $-\mathrm{Si}-\mathrm{CH}_{3}$ near $1255 \mathrm{~cm}^{-1}$ and $755 \mathrm{~cm}^{-1}$ indicate the existence of $\left(\mathrm{CH}_{3}\right)_{3} \mathrm{Si}$ - group.The stretching vibration band of $-\mathrm{Si}-\mathrm{CH}_{3}$ near $3000 \mathrm{~cm}^{-1}$ disappear.It shows that the trimethylchlorosilane which is called capping agent combined with polymers by hydrolysis-condensation reaction. The stretching vibration band of -Si-O-Si- near 
$1046 \mathrm{~cm}^{-1}$ indicates that the coating is an organic / inorganic hybrid system. The disappear of stretching vibration band of $-\mathrm{C}=\mathrm{C}-$ near $1630 \mathrm{~cm}^{-1}$ and $810 \mathrm{~cm}^{-}$ ${ }^{1}$ indicates that the polymerization reaction occurred in the double bond.

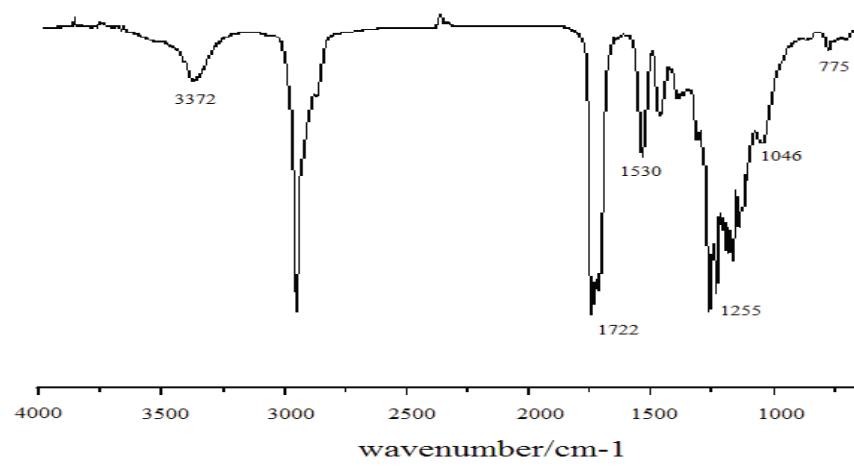

Fig. 3 FT-IR spectrum of hybrid coating

\subsection{Thermogravimetric analysis of hybrid coating}

The thermal stability of nano sol /PUA hybrid coating was analyzed by thermogravimetric analyzer. Nano sol and temperature have influence on the weightlessness of coating,as shown in Figure 4.The weightlessness of the coatings at $300^{\circ} \mathrm{C} \sim 450^{\circ} \mathrm{Cmainly}$ due to the thermal decomposition of the polyurethane acrylic polymer.Thermal decomposition would not happen when the temperature was below $300{ }^{\circ} \mathrm{C}$, thermal decomposition rate increased with temperature at $300^{\circ} \mathrm{C} \sim 450^{\circ} \mathrm{C}$, and the thermal decomposition was completed basically before $450^{\circ} \mathrm{C}$.Because of the decomposition of organic compounds in nano sol,the content of nano sol in coating were reduced from $15 \%$ and $20 \%$ to $12.4 \%$ and $17.2 \%$ after reaction.

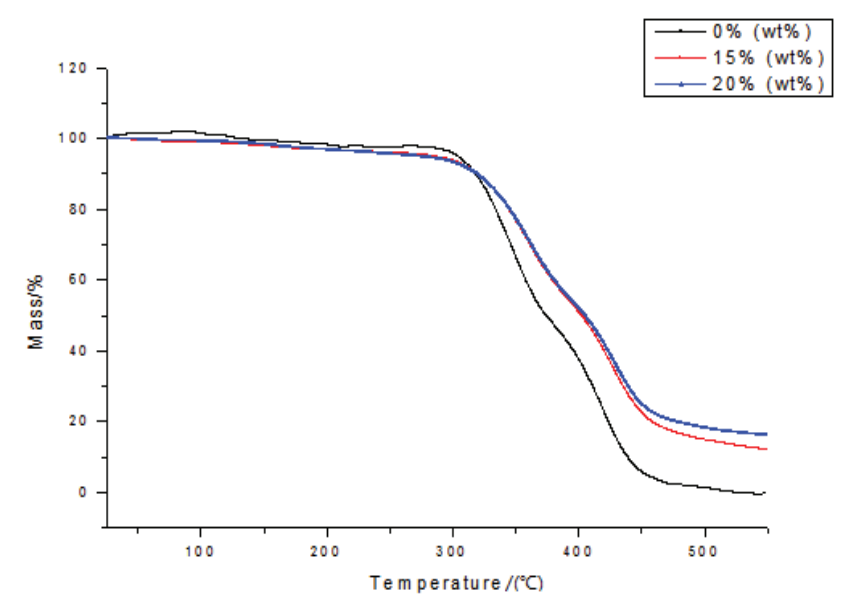

Fig. 4 The TGA analysis of nano-sol/PUA hybrid coating We also learn from the figure 4 that below $300^{\circ} \mathrm{C}$ the adding of the nano-sol has few effect on thethermal stability. The nano-sol plays an role in the effect of thermal stability when the temperature is over $300^{\circ} \mathrm{C}$. At $350^{\circ} \mathrm{C}$, the weightlessness of the hybrid films without adding nano sol has reached $35 \%$, and the weightlessness of hybrid films containing nano sol has just reached 19\%. It can be seen from the results that the addition of nano - sol can improve the thermal stability and heat resistance of PUA materials. This is mainly due to the nano particles in the sol and the organic polymers connected together though chemical bond to form interpenetrating crosslinked network, which increases the resistance to rotation, bending and moving of molecular chain and hinders the thermal decomposition of molecular chain.

\subsection{Pencil hardness of the filmlight cured nano hybrid materials}

PUA/nano sol hybrid film is a kind of organic / inorganic hybrid coating, and it is cured by photochemical reaction under UV irradiation. The UV curable organic / inorganic nano hybrid films were prepared by mixing the synthetic polyurethane acrylate resin and syntheticnano sol and joining the active monomer whose photoinitiator is type 1173.The pencil hardness of hybrid coating is $1 \mathrm{H}$ when the content of the nano sol is $10 \%$. The pencil hardness of hybrid coating has increased to $2 \mathrm{H}$ when the content of the nano sol is $15 \%$. The pencil hardness of hybrid coating increased to $3 \mathrm{H}$ when the content of the nano sol is $20 \%$. The organic/ inorganic phase structure in hybrid coating is shown in figure 5 , it is easy to learn from the figure that pencil hardness of hybrid coating increase with the increase of the content of nano sol. Sol molecules are combined with each other through the chemical bond. Inorganic phase and organic phase form the cross-linked network under the irradiation of the ultraviolet light. With the increase of the nano sol content, the proportion of inorganic phase in the films is increased, the reinforcing effect of inorganic phase in hybrid coating is also improved. As a result, the hardness of the hybrid films is increased with the increase of the content of the nano sol. Figure 6 is the microscopic model of hybrid coating . 


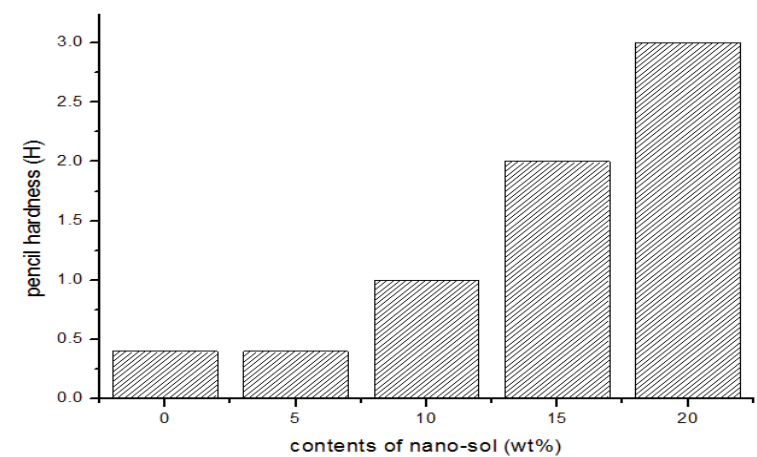

Fig. 5 Relationship between content of nano sol and pencil hardness of hybrid coating

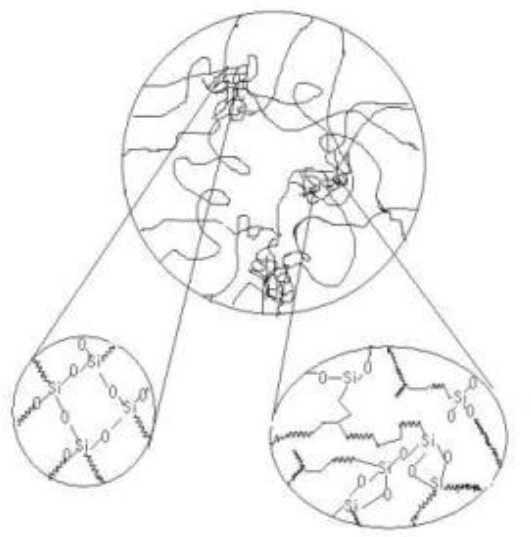

Fig. 6 The microscopic model of hybrid material

\section{References}

1. A.Endruweit; M,S,Johnson,A,C,Long Polym,Compos [J].2006,27,119

2. E.Lackey;K.Inamdar,L.Worrel;W.Al-

Akhdar;D.A.Wostrazky,RadTechReport[J].

$2001,15,36$

3. Jin Shuping, Liu Mingzhu, Chen Shilan, et al.Synthesis, characterization and the rapid response property of the temperature responsive PVP-gPNIPAM hydrogel[J].European Polymer Journal, 2008, 44: 2162-2170.

4. Zhang Jie, Chu Liangyin, Li Yuanke, et al.Dual thermo-and $\mathrm{pH}$-sensitive $\operatorname{poly}(\mathrm{N}-$ isopropylacrylamide-co-acrylic acid)hydrogels with rapid response behaviors[J].Polymer, 2007, 48: 17181728.

5. Liewen Liao, Zhengtang Liu, Xinming,Li. Solution properties of co-polymer hydrogel of AA/AMPS[J]. Advances in Chemical Engineering,2008,27(5):729732

6. Abd El-Rehim H A, Hegazy E A, Khalil F H, et
Figure 6 is the microscopic model of hybrid materials, inorganic phase of material is bonded to the resin in the form of chemical bond, chain in the inorganic phase containing photosensitive group and chain in the organic phase form interpenetrating network. The cross-linked network structure can improve the hardness of the material and change the mechanical properties of the material.

\section{Conclusions}

Polyester polyol, toluene diisocyanate (TDI) and Hydroxyethyl acrylate (HEA) were taken as raw material to synthesize polyurethane acrylate. UV curable organo silicon nano sol was synthesized by $\gamma$ methacryloxypropyltrime-thoxysilane. UV curable organic / inorganic nano hybrid materials were prepared by synthesized nano sol and polyurethane acrylate. Inorganic phase and organic phase form the cross-linked network under the irradiation of the ultraviolet light. The results showed that the heat resistance of the hybrid materials was increased with the increase of the amount of nano - sol and the hardness of the hybrid film was also increased.

al.Radiation preparation of drug carriers based polyacrylic acid(PAAc)using poly(vinyl pyrrolidone)(PVP)as a template polymer[J].Nuclear

7. ChangmeiKe, HouzhiWang, LilinLu, Preparation and properties of Polyacrylate / $\mathrm{TiO} 2-\mathrm{SiO} 2$ nanohybrid materials [J]. Glass just / composite, 2005 (4):23-26.

8. Wei Y,JinD, YangC.J Sol-Gel Sci Tech.1996, 7:191196 